\title{
4
}

\section{Insegurança Alimentar em famílias do Programa Bolsa Família}

\author{
Fernanda Witt ${ }^{1}$ Bianca Inês Etges ${ }^{2}$ e Luciano Lepper ${ }^{2}$
}

O objetivo deste estudo foi identificar a frequência de Insegurança Alimentar (IA) e verificar se existe associação entre IA com estado nutricional, número de integrantes do domicílio, sexo e escolaridade do responsável familiar de beneficiários do Programa Bolsa Família (PBF) no município de Vale do Sol (RS). Trata-se de um estudo transversal, com obtenção dos dados em 2017. Utilizou-se um questionário estruturado para obtenção de informações socioeconômicas e a Escala Brasileira de Insegurança Alimentar (EBIA) para medir a IA. O estado nutricional foi avaliado a partir dos indicadores baseados em peso e altura de acordo com a faixa de idade. Aplicouse o teste Qui-quadrado para avaliar a associação de IA com as variáveis selecionadas. A amostra foi constituída por 59 famílias. A frequência de IA foi de 78\%, apresentou-se associada ao índice peso/estatura (P/E) em crianças menores de 5 anos $(p=0,03)$, sendo a maior parte casos de grau leve. As variáveis avaliadas não se associaram com a IA com exceção do indicador P/E nas crianças menores de 5 anos. O elevado percentual de excesso de peso e obesidade encontrado nas famílias de baixa renda demonstra que a transição nutricional está presente nestas famílias. Destaca-se a necessidade de atuação efetiva do profissional da área de nutrição na Proteção Social Básica (PSB).

Palavras-chave: antropometria, estado nutricional, segurança alimentar.

\section{Food insecurity in households of the bolsa familia program}

The goal of this study has been to identify the prevalence of Food Insecurity (IA) and check if there is a correlation between with nutritional status, number of household members, sex and education of the main recipient of the Bolsa Familia Program (PBF) in the municipality of Vale do Sol (RS). This is a cross-sectional study of data obtained in 2017. A structured questionnaire was used for obtaining socioeconomic information and the Brazilian Food Insecurity Scale (EBIA) to measure the IA. Nutritional status was evaluated through the data obtained in the measurements of weight and height according to age. The Chi-squared test was used to evaluate the Association of IA with the variables. The sample was of 59 families. The IA frequence was $78 \%$, associated with the index weight/height $(\mathrm{W} / \mathrm{H})$ in children under 5 years $(\mathrm{p}=0.03)$, being the most only mild cases. The evaluated variables are not related, but the W/H indicator in children under 5 years. The high percentage of overweightness and obesity found in the study shows that the nutritional transition is occurring in low-income families. The need for a professional nutrition in Basic Social Protection (PSB) has been observed.

Keywords: anthropometry, nutritional status, food security.

${ }^{1}$ Departamento de Educação Física e Saúde. Universidade de Santa Cruz do Sul. Endereço para correspondência: Av. Independência no 2.293 - Bairro Universitário, Santa Cruz do Sul - RS, CEP: 96815-900. Tel. Celular: (51) 9951-26725.E-mail: fernandawitt@outlook.com

2 Departamento de Educação Física e Saúde. Universidade de Santa Cruz do Sul. 


\section{INTRODUÇÃO}

Em 2003 foi criado pelo governo federal brasileiro o Programa Bolsa Família (PBF), sendo este um programa de transferência de renda com o propósito de proporcionar o acesso das famílias aos direitos sociais básicos e acabar com a pobreza por meio das condicionalidades, que são as obrigações assumidas pelas famílias beneficiárias assim como pelo poder público[1].

$\mathrm{O}$ PBF auxilia no combate à pobreza de duas maneiras: transferindo mensalmente um valor em dinheiro de forma direta às famílias; fazendo acompanhamento, na área de saúde, com crianças, adolescentes e gestantes, e na educação com as crianças e os adolescentes que fazem parte do programa ${ }^{[2]}$. Esse programa destina-se às famílias em situação de pobreza e de extrema pobreza e tem o intuito de promover a Segurança Alimentar e Nutricional (SAN) dos seus integrantes e de reduzir as desigualdades sociais do país. Para definição da pobreza e extrema pobreza é usado um limite de renda, podendo assim participar do programa todas as famílias com renda per capita de até $\mathrm{R} \$ 85,00$ mensais (extrema pobreza); famílias com renda per capita entre $\mathrm{R} \$ 85,01$ e $\mathrm{R} \$ 170,00$ mensais (pobreza), desde que entre os membros tenham crianças ou adolescentes de 0 a 17 anos [2].

A SAN é definida no artigo 3o da Lei Orgânica de Segurança Alimentar e Nutricional (LOSAN) no 11.346 de 15 de setembro de 2006, como um direito de todos a uma alimentação adequada com acesso frequente e permanente a alimentos de qualidade, em quantidade satisfatória, sem afetar o acesso a outras necessidades fundamentais, baseando-se em práticas alimentares promotoras de saúde que respeitem a diversidade cultural e que sejam ambiental, cultural, econômica e socialmente sustentáveis ${ }^{[3]}$.

A Emenda Constitucional no 64, aprovada em 4 de fevereiro de 2010, introduziu o direito humano à alimentação e o acesso aos alimentos como um direito social no artigo 6o da Constituição Federal: "São direitos sociais a educação, a saúde, a alimentação, o trabalho, a moradia, o lazer, a segurança, a previdência social, a proteção à maternidade e à infância, a assistência aos desamparados, na forma desta Constituição" [4]. A inserção desse direito na listagem dos direitos sociais trouxe para os cidadãos brasileiros melhores condições de vida, pois o Estado (governo) tornou-se obrigado, a garantir a todos, não apenas o direito à alimentação, mas sim a uma alimentação de qualidade ${ }^{[5]}$.

A prioridade política determinada para $\mathrm{o}$ combate à fome e à miséria nos últimos anos tem ocasionado a melhora significativa das condições sociais da população brasileira, produzindo resultados positivos na SAN. Os resultados obtidos são devidos a um conjunto de medidas adotados por diversos setores, como o aumento do poder de compra do salário mínimo, o crescimento do acesso ao emprego, o fortalecimento dos programas de transferência de renda às famílias de baixa renda, a consolidação dos sistemas universais de educação e saúde, a melhoria do Programa Nacional de Alimentação Escolar (PNAE) e a consolidação dos programas de agricultura familiar no país (Programa de Aquisição de Alimentos - PAA). Isso se confirma no relatório global da Organização das Nações Unidas para Alimentação e Agricultura $(\mathrm{FAO})^{[6]}$ com a informação de que o Brasil deixou o mapa mundial da fome em 2014.

Por sua vez a Insegurança Alimentar (IA) retrata a limitação de acesso a alimentos em quantidades suficientes para satisfazer as necessidades básicas, impactando a saúde em diversos aspectos[7]. É um problema com diversas dimensões que se caracteriza como uma dificuldade de saúde mundial e está relacionada de forma direta com a luta pelo Direito Humano à Alimentação Adequada (DHAA) ${ }^{[8]}$. Existem vários fatores relacionados a $\mathrm{IA}$, entre eles os socioeconômicos, demográficos, condições de moradia e saneamento, disponibilidade e consumo de alimentos, e participação em programas sociais do governo[8,9,10].

Em meados da década de 1990 foi elaborado, nos Estados Unidos, um método para avaliar a IA em seus diferentes níveis conforme o avanço de sua gravidade (leve, moderada e grave). No Brasil esse método é chamado de Escala Brasileira de Insegurança Alimentar (EBIA), que avalia a IA por meio da compreensão das famílias sobre o acesso aos alimentos ${ }^{[11]}$. Com a EBIA, a IA é constatada em seus diversos níveis, começando pela preocupação da falta do alimento no domicílio antes que se tenha dinheiro para comprar novamente, chegando a uma condição em que a família passa por tempos de limitação na acessibilidade de alimentos a suas familias ${ }^{[0]}$. Para Santos ${ }^{[]]}$é importante investigar o efeito da IA para a família, assim como as alterações que podem ser 
causadas individualmente, acometendo o estado nutricional dos seus membros.

Nesse contexto, objetivou-se com o presente estudo identificar a frequência de IA e verificar a existência de correlação entre o grau de IA com estado nutricional, número de integrantes do domićlío, sexo e escolaridade do responsável familiar de beneficiários do PBF no município de Vale do Sol (RS).

\section{MÉTODO}

Trata-se de um estudo transversal, de natureza quantitativa, realizado entre os meses de março e abril de 2017, com famílias beneficiárias do PBF, participantes das oficinas do Serviço de Proteção e Atendimento Integral à Família (PAIF), realizadas mensalmente em 10 localidades do município de Vale do Sol (RS), Brasil, com população estimada de 11.077 habitantes. A pesquisa foi realizada após aprovação do Comitê de Ética em Pesquisa da Universidade de Santa Cruz do Sul (UNISC) sob o número CAEE 64831416.9.0000.5343, parecer 1.942.261 e mediante autorização da coordenadora responsável pelas oficinas do PAIF.

Foram incluídas na pesquisa famílias em que os responsáveis familiares, maiores de 18 anos, se fizessem presentes nas oficinas do PAIF, para aplicação do questionário da EBIA e assinatura do Termo de Consentimento Livre e Esclarecido (TCLE). Também foram critérios de inclusão as famílias serem beneficiárias do PBF, terem cadastro único no município de Vale do Sol, todos os membros das famílias maiores de 18 anos assinarem o TCLE e todos os membros menores de 18 anos assinarem o Termo de Assentimento Livre e Esclarecido (TALE) e seus pais ou responsáveis assinarem o TCLE.

No primeiro encontro foram recolhidas assinaturas do TCLE, aplicado um questionário socioeconômico e a EBIA de forma individual com os responsáveis familiares presentes na oficina do PAIF. Os dados socioeconômicos e demográficos investigados foram: sexo e escolaridade do responsável familiar, sexo dos demais integrantes, renda per capita, número de moradores por domicílio, número de cômodos no domicílio, local do domicílio, tipo de domicilio, abastecimento de água, escoamento de esgoto, destino do lixo e forma de iluminação. A EBIA conta com 14 perguntas e investiga o acesso das famílias à alimentação nos últimos três meses para caracterização da situação de segurança ou IA em seus diferentes níveis. Sua análise é baseada em um gradiente de pontuação final resultante do somatório das respostas afirmativas de 14 questões, sendo essa pontuação classificada em duas maneiras diferentes, uma para domicílios com menores de 18 anos e outra para sem menores de 18 anos. Nos domicilios com menores, segurança alimentar (nenhuma restrição alimentar e preocupações futuras com a falta de alimentos) equivale a 0 ponto, IA leve (preocupação ou incerteza quanto ao acesso aos alimentos) de 1 a 5 pontos, IA moderada (restrições quantitativas especialmente relevantes entre pessoas adultas) de 6 a 9 pontos e a IA grave (redução importante da quantidade de alimentos disponíveis, tanto para a alimentação de adultos, como para a de crianças) de 10 a 14 pontos. Já nos domicílios sem menores de 18 anos, segurança alimentar também equivale a 0 ponto, IA leve de 1 a 3 pontos, IA moderada de 4 a 5 pontos e a IA grave de 6 a 8 pontos ${ }^{[12]}$.

No segundo encontro, realizado nos domicílios, os Agentes Comunitários de Saúde (ACSs) realizaram a avaliação antropométrica dos membros das famílias, após treinamento prévio, utilizando uma balança digital da marca Zeex Corpore ${ }^{\circledR}$ com carga máxima de $150 \mathrm{~kg}$ e para a aferição da estatura, foi utilizada uma fita não flexível e inelástica de $150 \mathrm{~cm}$. Para aferição do peso de crianças com menos de dois anos, um responsável pela criança foi pesado inicialmente e anotado o resultado obtido. Em seguida o mesmo responsável foi pesado segurando a criança a ser avaliada no colo, descontou-se o peso inicial do responsável, obtendo assim o resultado que correspondeu ao peso da criança. A estatura destas crianças foi obtida por meio de um antropômetro infantil com $100 \mathrm{~cm}$ de comprimento. Os ACSs também realizaram o recolhimento de assinaturas dos TCLEs e TALEs de integrantes que não estavam presentes nas oficinas do PAIF.

Os dados antropométricos, bem como o sexo e a idade, possibilitaram a realização da avaliação do estado nutricional dos membros das famílias. Os indicadores Índice de Massa Corporal para Idade (IMC/I), Peso para Idade (P/I), Peso para Estatura $(\mathrm{P} / \mathrm{E})$ e Estatura para Idade (E/I) foram utilizados para crianças menores de 5 anos; IMC/I, P/I e E/I para crianças de 5 a 10 anos; IMC/I e E/I para adolescentes entre 10 e 19 anos de idade. Todos os índices foram 
classificados segundo os protocolos do Sistema de Vigilância Alimentar e Nutricional (SISVAN) ${ }^{[13]}$ a partir das curvas de crescimento da Organização Mundial de Saúde (OMS) [14,15]. Para a avaliação da população adulta foi calculado e classificado o IMC[16].

Os dados foram tabulados no software Microsoft Excel (2013) e analisados no programa Statistical Package for Social Sciences (versão 20.0). Realizouse uma análise descritiva para caracterização da amostra e empregou-se o teste de Qui-quadrado $\left(\chi^{2}\right)$ para comparação do nível de IA, estado nutricional, número de membros do domicílio, sexo e escolaridade do chefe familiar. Todos os dados foram verificados quanto à normalidade e homocedasticidade e considerou-se nível de significância de $p<0,05$.

\section{RESULTADOS}

Esta pesquisa contou com 59 famílias, o que corresponde a $19,0 \%$ do total de familias beneficiárias no município em abril de 2017. Das 59 famílias estudadas, a média de integrantes por família foi 4,14 \pm 1,36 (mínimo 2 e máximo 8), destas, 49,1\% tinham entre 4-6 membros, com renda per capita média de $\mathrm{R} \$ 141,14 \pm 80,30$ (mínimo $\mathrm{R} \$ 27,00$ e máximo $\mathrm{R} \$ 312,00)$ (Tabela 1).

Tabela 1. Distribuição de uma amostra de famílias beneficiárias do PBF de acordo com características socioeconômicas e demográficas. Vale do Sol, RS, Brasil, 2017

\begin{tabular}{|c|c|c|c|}
\hline \multirow{2}{*}{ Variável } & \multirow{2}{*}{ Média $\pm D P$} & \multicolumn{2}{|c|}{ Frequência } \\
\hline & & $\mathrm{n}$ & $\%$ \\
\hline Integrantes por família & $4,14 \pm 1,36$ & & \\
\hline \multicolumn{4}{|l|}{ Classificação membros } \\
\hline 1-3 membros & & 26 & 44,1 \\
\hline 4-6 membros & & 29 & 49,1 \\
\hline$>6$ membros & & 4 & 6,8 \\
\hline Idade & $24,55 \pm 16,48$ & & \\
\hline \multicolumn{4}{|l|}{ Sexo } \\
\hline Feminino & & 134 & 54,9 \\
\hline Masculino & & 110 & 45,1 \\
\hline \multicolumn{4}{|l|}{ Escolaridade do chefe da família } \\
\hline Analfabeto & & 3 & 5,1 \\
\hline Ensino fundamental incompleto & & 50 & 84,7 \\
\hline Ensino fundamental completo & & 4 & 6,8 \\
\hline Ensino médio incompleto & & 2 & 3,4 \\
\hline \multicolumn{4}{|l|}{ Sexo Chefe de família } \\
\hline Feminino com companheiro & & 47 & 79,7 \\
\hline Feminino - solteira & & 11 & 18,6 \\
\hline Masculino - solteiro & & 1 & 1,7 \\
\hline Renda per capita (Reais) & $141,14 \pm 80,30$ & & \\
\hline Número de cômodos & $4,80 \pm 1,19$ & & \\
\hline \multicolumn{4}{|l|}{ Local de domicilio } \\
\hline Rural & & 58 & 98,3 \\
\hline Urbano & & 1 & 1,7 \\
\hline \multicolumn{4}{|l|}{ Tipo de domicilio } \\
\hline Alugada & & 2 & 3,4 \\
\hline Cedida & & 41 & 69,5 \\
\hline Própria & & 16 & 27,1 \\
\hline \multicolumn{4}{|l|}{ Abastecimento de água } \\
\hline Rede pública & & 34 & 57,6 \\
\hline Nascente & & 3 & 5,1 \\
\hline Poço artesiano & & 22 & 37,3 \\
\hline \multicolumn{4}{|l|}{ Escoamento de Esgoto } \\
\hline Fossa séptica & & 53 & 89,8 \\
\hline Vala a céu aberto & & 3 & 5,1 \\
\hline Outra forma & & 3 & 5,1 \\
\hline \multicolumn{4}{|l|}{ Lixo } \\
\hline Coletado diretamente & & 46 & 78,0 \\
\hline Queimado/enterrado & & 13 & 22,0 \\
\hline \multicolumn{4}{|l|}{ Iluminação } \\
\hline Medidor próprio & & 43 & 72,9 \\
\hline Medidor comunitário & & 16 & 27,1 \\
\hline \multicolumn{4}{|l|}{ Classificação EBIA } \\
\hline Segurança alimentar & & 13 & 22,0 \\
\hline Insegurança alimentar & & 46 & 78,0 \\
\hline Insegurança alimentar leve & & 41 & 89,1 \\
\hline Insegurança alimentar moderada & & 5 & 10,9 \\
\hline
\end{tabular}


Em relação ao sexo do responsável familiar, 98,3\% eram do sexo feminino, destas, $79,7 \%$, possuem companheiro. Quanto aos dados socioeconômicos, $84,7 \%$ dos responsáveis familiares tem ensino fundamental incompleto. A maioria das famílias reside na zona rural $(98,3 \%)$ em domicílios cedidos $(69,5 \%)$, com média de 4,80 $\pm 1,19$ cômodos (mínimo 2 e máximo 8). Com relação às condições do saneamento básico, $78 \%$ das famílias residem em áreas com coleta pública de lixo, 89,8\% tem escoamento do esgoto com fossa séptica, 57,6\% tem acesso ao abastecimento de água da rede pública e $72,9 \%$ tem iluminação com medidor próprio. De acordo com a classificação da EBIA, 22\% das famílias apresentam Segurança
Alimentar e 78\% apresentam IA, destas a maioria em situação de IA leve (89,1\%) (Tabela 1).

Em relação aos indicadores IMC/I, P/I e E/I e a classificação da EBIA não foi observada associação significativa, mostrando que existe uma distribuição normal do estado nutricional com o grau de segurança e IA. Já em relação à classificação de $\mathrm{P} / \mathrm{E}$ observou-se associação significativa $(p=0,03)$ com a classificação da EBIA, indicando que a IA está associada com o risco de sobrepeso, sobrepeso e obesidade em crianças menores de 5 anos (Tabela 2).

Tabela 2. Relação da classificação da EBIA com IMC/I, P/I, P/E e E/I de crianças com menos de 5 anos, integrantes das famílias beneficiárias do PBF. Vale do Sol, RS, Brasil, 2017 (n=20)

\begin{tabular}{|c|c|c|c|c|c|}
\hline & \multicolumn{5}{|c|}{ EBIA-Classificação } \\
\hline & \multicolumn{2}{|c|}{ Segurança alimentar } & \multicolumn{2}{|c|}{ Insegurança alimentar } & \multirow{2}{*}{$\boldsymbol{P}$} \\
\hline & $\mathrm{n}$ & $\%$ & $\mathbf{n}$ & $\%$ & \\
\hline IMC/I & & & & & 0,064 \\
\hline Magreza & 0 & 0,0 & 1 & 5,0 & \\
\hline Eutrofia & 2 & 10,0 & 2 & 10,0 & \\
\hline Risco de sobrepeso & 0 & 0,0 & 10 & 50,0 & \\
\hline Sobrepeso & 0 & 0,0 & 3 & 15,0 & \\
\hline Obesidade & 0 & 0,0 & 2 & 10,0 & \\
\hline $\mathrm{P} / \mathrm{I}$ & & & & & 0,619 \\
\hline Peso adequado para idade & 2 & 10,0 & 16 & 80,0 & \\
\hline Peso elevado para idade & 0 & 0,0 & 2 & 10,0 & \\
\hline $\mathbf{P} / \mathbf{E}$ & & & & & 0,031 \\
\hline Eutrofia & 2 & 10,0 & 2 & 10,0 & \\
\hline Risco de sobrepeso & 0 & 0,0 & 11 & 55,0 & \\
\hline Sobrepeso & 0 & 0,0 & 3 & 15,0 & \\
\hline Obesidade & 0 & 0,0 & 2 & 10,0 & \\
\hline E/I & & & & & 0,822 \\
\hline Muita baixa estatura para idade & 0 & 0,0 & 1 & 5,0 & \\
\hline Baixa estatura para idade & 0 & 0,0 & 2 & 10,0 & \\
\hline Estatura adequada para idade & 2 & 10,0 & 15 & 75,0 & \\
\hline
\end{tabular}

A maioria das crianças entre 5 e 10 anos, dos adolescentes (entre 10,1 e 18 anos) e dos adultos estão em situação de IA $(76,0 \%, 77,9 \%, \quad 74,6 \%$, respectivamente). Segundo classificação de IMC/I a frequência de excesso de peso foi elevada nessas faixas etárias $(20,0 \%, 11,7 \%, 27,0 \%$, respectivamente) e a obesidade foi maior na população adulta $(31,2 \%)$. A eutrofia prevaleceu nos adolescentes $(80,5 \%)$ e os índices de magreza/baixo peso foram superiores nas crianças de 5-10 anos (12,0\%). Não foi observada associação estatisticamente significativa da classificação do estado nutricional com a classificação da EBIA em nenhuma destas três faixas etárias.
A Tabela 3 apresenta a associação da IA com número de moradores do domićlí, sexo e escolaridade do chefe familiar. Entre as familias entrevistadas, todas que tinham mais de 6 moradores ficaram em IA, nas que tinham dois chefes familiares o índice de segurança alimentar foi maior e todas aquelas em que o chefe familiar era analfabeto apresentaram IA. Não foram encontradas associações estatisticamente significativas $(p<0,05)$. 
Tabela 3. Distribuição das famílias beneficiárias do PBF de acordo com variáveis socioeconômicas e situação de segurança e insegurança alimentar no município de Vale do Sol, RS. Brasil, 2017

\begin{tabular}{|c|c|c|c|c|c|}
\hline & \multicolumn{5}{|c|}{ EBIA - Classificação } \\
\hline & \multicolumn{2}{|c|}{ Segurança alimentar } & \multicolumn{2}{|c|}{ Insegurança alimentar } & \multirow{2}{*}{$P$} \\
\hline & $\mathrm{n}$ & $\%$ & $\mathrm{n}$ & $\%$ & \\
\hline Número de moradores no domicílio & & & & & 0,574 \\
\hline 1-3 membros & 5 & 8,5 & 21 & 35,6 & \\
\hline 4-6 membros & 8 & 13,5 & 21 & 35,6 & \\
\hline$>6$ membros & 0 & 0,0 & 4 & 6,8 & \\
\hline Sexo responsável familiar & & & & & 0,760 \\
\hline Feminino - solteira & 1 & 1,7 & 10 & 17,0 & \\
\hline Masculino - solteiro & 0 & 0,0 & 1 & 1,7 & \\
\hline Feminino com companheiro & 12 & 20,3 & 35 & 59,3 & \\
\hline Escolaridade responsável familiar & & & & & 0,154 \\
\hline Analfabeto & 0 & 0,0 & 3 & 5,1 & \\
\hline Ensino fundamental incompleto & 12 & 20,3 & 38 & 64,4 & \\
\hline Ensino fundamental completo & 0 & 0,0 & 4 & 6,8 & \\
\hline Ensino médio incompleto & 1 & 1,7 & 1 & 1,7 & \\
\hline
\end{tabular}

\section{DISCUSSÃO}

No presente estudo constatou-se elevada proporção de famílias do $\mathrm{PBF}$ em situação de IA $(78,0 \%)$, sendo a maior parte classificada em IA leve (89,1\%). Dados semelhantes aos encontrados neste estudo para a situação de IA também foram observados em um estudo realizado com 150 famílias beneficiárias do PBF de um município do norte de Minas Gerais ${ }^{[17]}$, em que a maioria dos entrevistados vivenciava IA $(72,0 \%)$, sendo a maior proporção classificada com IA leve $(50,0 \%)$. Na pesquisa de Santos e Silva ${ }^{[18]}$ realizada em 200 domicílios de beneficiários do PBF no município de Itabuna (BA), verificou-se frequência de IA em $83,0 \%$, prevalecendo a IA moderada $(33,0 \%)$. No estudo realizado com 421 famílias beneficiárias do PBF, no município de Toledo (PR) ${ }^{[19]}$, constatou-se a presença de IA em $74,6 \%$, sendo a maioria classificada com IA leve $(44,9 \%)$.

$\mathrm{Na}$ pesquisa realizada pelo Instituto Brasileiro de Análises Sociais e Econômicas (IBASE) [20], com 5 mil titulares do cartão bolsa família, em 229 municípios brasileiros, $83,0 \%$ das famílias encontravam-se em situação de IA e 17,0\% em segurança alimentar. As predominâncias de IA constatadas foram bem mais elevadas do que as encontradas pelo Instituto Brasileiro de Geografia e Estatística (IBGE) no ano de 2013[21], em pesquisa feita em todo o território nacional. No entanto, deve-se ponderar que o artigo em questão trabalhou com amostra específica, composta de familias beneficiárias do PBF.
No tocante ao excesso de peso e obesidade foram mais frequentes que a desnutrição na maioria das faixas etárias das famílias em IA. O déficit de peso em relação à estatura também não foi verificado em crianças até cinco anos de idade, 15,0\% apresentavam déficit de estatura e 15,0\% sobrepeso. Resultados semelhantes foram encontrados na pesquisa realizada com 189 crianças menores de cinco anos no município de João Câmara (RN) ${ }^{[22]}$, a qual verificou que 9,9\% das crianças apresentaram déficit de estatura e 14,0\% excesso de peso. A transição nutricional vem sendo retratada em todas as regiões brasileiras e em todos os grupos de renda ${ }^{[23]}$.

No estudo realizado na área urbana de Pelotas (RS) ${ }^{[9]}$ com 132 famílias em situação de IA também não foi observado o déficit de peso em relação à estatura em crianças até cinco anos de idade, contudo, o déficit em estatura foi de $21,0 \%$ e do excesso de peso foi de 3,0\%. Carvalho et al.[24] revelam a partir da avaliação nutricional que em crianças menores de cinco anos o processo de transição nutricional é visível, com baixas prevalências de déficit de peso para idade e prevalências mais altas de excesso de peso.

Ao avaliar o estado nutricional dos integrantes das famílias os resultados do presente estudo estão semelhantes ao estudo realizado em Pelotas (RS)[?], o qual demonstrou que enquanto o déficit nutricional não foi predominante nos diferentes grupos populacionais, a prevalência de obesidade foi mais elevada especialmente em adultos $(23,0 \%)$. 
De acordo com a pesquisa Vigilância de Fatores de Risco e Proteção para Doenças Crônicas por Inquérito Telefônico (VIGITEL)[25] e a Pesquisa Nacional de Saúde (PNS)[26], realizadas com a população brasileira de 18 anos ou mais de idade, 52,3\% e $56,9 \%$ encontram-se com excesso de peso e $17,0 \%$ e $20,8 \%$ com obesidade, respectivamente. O déficit nutricional não foi predominante na PNS assim como neste estudo.

Em relação ao número de integrantes no domicílio, a presente pesquisa constatou que as famílias com mais de 6 membros aumentaram a chance de apresentar IA, semelhante ao estudo realizado com beneficiários do PBF em Viçosa (MG) ${ }^{[27]}$, em que foram encontradas maiores prevalências de IA em domicílios que abrigavam 4 ou mais moradores $(73,1 \%)$. Já estudo realizado com beneficiários de Toledo (PR) ${ }^{[19]}$ constatou que famílias com 5 membros ou mais aumentaram a chance de passar por IA, quando comparadas as famílias com menor número de membros.

$\mathrm{Na}$ análise socioeconômica, a maioria dos entrevistados afirmou ter um cônjuge $(79,6 \%)$, considerando ambos como chefe familiar. Observou-se também que as famílias com apenas o responsável familiar do sexo feminino teve maiores percentuais de IA $(90,9 \%)$. No estudo realizado com 168 famílias de beneficiários do PBF em Colombo (PR) ${ }^{[28]}$ a IA foi predominante nos domicílios em que o chefe da família era do sexo feminino $(75,6 \%)$. Na pesquisa realizada com famílias beneficiárias de Maceió (AL) ${ }^{[29]}$ as proporções de IA foram maiores nas famílias que a mãe era responsável $(91,9 \%)$.

Em relação à escolaridade, a maioria dos chefes familiares tem baixo grau de escolaridade e $89,8 \%$ tem o ensino fundamental incompleto, confirmando-se este perfil em outros estudos. No estudo realizado com famílias beneficiárias em Araraquara (SP) ${ }^{[12]}$ verificou-se que $67,0 \%$ dos titulares do PBF têm o ensino fundamental. Já no estudo com beneficiários de Itabuna (BA) ${ }^{[18]}$, observou-se que a maioria dos titulares sabe ler e escrever, mas o grau de escolaridade ainda é baixo, pois $65,5 \%$ não concluíram o ensino fundamental e apenas 19,5\% concluíram o ensino médio. Segundo o IBASE (2008) [20] essa é uma consequência negativa, sendo que a ausência de qualificação profissional causa baixos rendimentos e instáveis condições de trabalho.

\section{CONCLUSÃO}

A IA foi predominante nas famílias beneficiárias do PBF no município de Vale do Sol, sendo a maior parte casos de grau leve. As variáveis avaliadas (estado nutricional, número de membros da família, sexo e escolaridade do chefe familiar) não se associaram a IA com exceção do indicador $\mathrm{P} / \mathrm{E}$ nas crianças menores de 5 anos.

O elevado percentual de excesso de peso e obesidade encontrado nessa população reforça o que estudos que demonstram que a transição nutricional está acontecendo nas famílias de baixa renda, provavelmente decorrente de consumo excessivo de alimentos industrializados, com alto valor calórico e baixo custo.

Observa-se a necessidade de um profissional da área de nutrição na Proteção Social Básica (PSB), pois esse profissional tem a capacidade de assessorar esta população a realizar o uso correto dos baixos recursos que recebem na compra de alimentos com melhor qualidade nutricional e menor custo.

\section{REFERÊNCIAS}

[1] Brasil. O estado da segurança alimentar e nutricional no Brasil: um retrato multidimensional. Relatório 2014. Brasilia: FAO; 2014.

[2] Brasil. Ministério do Desenvolvimento Social e Agrário. Bolsa Família [internet]. 2016 [acesso em 09 out 2016]. Disponível em: http://mds.gov.br/assuntos/bolsafamilia/o-que-e/como-funciona

[3] Brasil. Lei no 11.346, de 15 de setembro de 2006. Cria o Sistema Nacional de Segurança Alimentar e Nutricional SISAN com vistas em assegurar o direito humano à alimentação adequada e dá outras providências [internet]. 2006 [acesso em 10 nov 2016]. Disponível em: https:/ / www.planalto.gov.br

[4] Brasil. Emenda Constitucional no 64, de 4 de fevereiro de 2010. Altera o art. 6o da Constituição Federal, para introduzir a alimentação como direito social [internet]. 2010 [acesso em 10 nov 2016]. Disponível em: http://www.planalto.gov.br

[5] Lisboa RC. Direito humano à alimentação adequada. Revista Eletrônica de Direito do Centro Universitário Newton Paiva. 2013:365-374. 
[6] Brasil. Ministério do Desenvolvimento Social e Combate à Fome. Secretaria de Avaliação e Gestão da Informação. Secretaria Nacional de Segurança Alimentar e Nutricional. MAPASAN 2014: Mapeamento de Segurança Alimentar e Nutricional. Brasília(DF); 2015.

[7] Carmichael SL, Yang W, Herring A, Abrams B, Shaw GM. Maternal food insecurity is associated with increased risk of certain birth defects. The Journal of Nutrition. 2007;9(137):2087-2092.

[8] Guerra LDS, Espinosa MM, Bezerra ACD, Guimarães LV, Lima-Lopes MA. Insegurança alimentar em domicílios com adolescentes da Amazônia Legal Brasileira: prevalência e fatores associados. Cad. Saúde Pública [internet]. 2013 [acesso em 26 maio 2017];29(2):335-348. Disponível em: http://www.scielo.br/scielo

[9] Santos JV, Gigante DP, Domingues MR. Prevalência de insegurança alimentar em Pelotas, Rio Grande do Sul, Brasil, e estado nutricional de indivíduos que vivem nessa condição. Cad. Saúde Pública [internet]. 2010;26(1):41-49. Disponível em: http://www.scielo.br/scielo

[10] Oliveira JS, Lira PIC, Veras ICL, Maia SR, Lemos MCC, Andrade SLLS, et al. Estado nutricional e insegurança alimentar de adolescentes e adultos em duas localidades de baixo índice de desenvolvimento humano. Rev. Nutr. [internet]. 2009 [acesso em 26 maio 2017];22(4):453-465. Disponível em: http://www.scielo.br/scielo

[11] Pérez-Escamilla R, Segall-Corrêa AM, Maranha LK, Sampaio MFA, Marin-León L, Panigassi G. An adapted version of the U.S. Department of Agriculture Food Insecurity module is a valid tool for assessing household food insecurity in Campinas, Brazil. J. Nutr. 2004;8134(8):1923-8.

[12] Traldi DRC, Almeida LMMC, Ferrante VLSB. Repercussões do Programa Bolsa Família no município de Araraquara, SP: um olhar sobre a segurança alimentar e nutricional dos beneficiários. Interações [internet]. 2012 [acesso em 26 maio 2017];13(1):23-37. Disponível em: http://www.scielo.br/scielo

[13] Ministério da Saúde. Protocolos do Sistema de Vigilância Alimentar e Nutricional (Sisvan) na assistência à saúde. Brasilia(DF); 2008.

[14] World Health Organization. WHO Child Growth Standards: length/height-for-age, weight-for-age, weight-forleigth, weight-for-height and body mass index-for-age. Methods and development. WHO (nonserial publication). Geneva: WHO; 2006.
[15] World Health Organization. Development of a WHO growth reference for school-aged children and adolescents. Bull World Health Organ. 2007;7:85-660.

[16] World Health Organization. Physical Status: The Use and Interpretation of Anthropometric Indicators of Nutritional Status. Geneva; 1995. (WHO Technical Report Series, 854).

[17] Nunes TS, Cruz JMG, Pinho L. Avaliação da segurança alimentar e nutricional de famílias beneficiárias do Programa Bolsa Família. Nutrire [internet]. 2014 [acesso em 26 maio 2017];2(39):233-242. Disponível em: http://docs.bvsalud.org/biblioref/2017/05/322136/artigo. pdf

[18] Santos CF, Silva MS. Segurança alimentar e programa bolsa família no município de Itabuna - Bahia. In: Anais da IV Semana do Economista e IV Encontro de Egressos; 2014; Itabuna. Itabuna; 2014.

[19] Anschau FR, Matsuo T, Segall-Corrêa AM. Insegurança alimentar entre beneficiários de programas de transferência de renda. Rev. Nutr. [internet]. 2012 [acesso em 26 maio 2017];25(2):177-189.

Disponível

em:

http://www.scielo.br/scielo

[20] Instituto Brasileiro de Análises Sociais e Econômicas. Repercussões do Programa Bolsa Família na segurança alimentar e nutricional das famílias beneficiadas [internet]. 2008 [acesso em 26 maio 2017]. Disponível em: www.ibase.br

[21] Instituto Brasileiro de Geografia e Estatística. Pesquisa Nacional por Amostra de Domicílios: Segurança Alimentar 2013 - Suplemento [internet]. 2013 [acesso em 26 maio 2017]. Disponível em: http://www.ibge.gov.br

[22] Saldiva SRDM, Silva LFF, Saldiva PHN. Avaliação antropométrica e consumo alimentar em crianças menores de cinco anos residentes em um município da região do semiárido nordestino com cobertura parcial do programa bolsa família. Rev. Nutr. [internet]. 2010 [acesso em 26 maio 2017];23(2):221-229. Disponível em: http:/ /www.scielo.br

[23] IBGE. Instituto Brasileiro de Geografia e Estatística. Pesquisa de Orçamentos Familiares 2008-2009: antropometria e estado nutricional de crianças, adolescentes e adultos no Brasil [internet]. 2010 [acesso em 26 maio 2017]. Disponível em: biblioteca.ibge.gov.br

[24] Carvalho AT, Almeida ER, Nilson EAF, Ubarana JA, Fernández IM, Immink M. Métodos de análise em programas de segurança alimentar e nutricional: uma experiência no Brasil. Ciênc. Saúde Coletiva [internet]. 2013 [acesso em 26 
maio 2017];18(2):309-321. Disponível em: http://www.scielo.br/scielo

[25] Brasil. Ministério da Saúde. Vigitel Brasil 2015 Saúde Suplementar: vigilância de fatores de risco e proteção para doenças crônicas por inquérito telefônico [recurso eletrônico]. Ministério da Saúde, Agência Nacional de Saúde Suplementar: Brasília(DF); 2017.

[26] IBGE. Instituto Brasileiro de Geografia e Estatística. Pesquisa Nacional de Saúde, 2013. Rio de Janeiro: IBGE;2014.

[27] Souza NN, Dias MM, Sperandio N, Franceschini SCC, Priore SE. Perfil socioeconômico e insegurança alimentar e nutricional de famílias beneficiárias do Programa Bolsa Família no município de Viçosa, Estado de Minas Gerais, Brasil, em 2011: um estudo epidemiológico transversal. Epidemiol. [internet]. 2012 [acesso em 26 maio 2017];21(4):655-662. Disponível em: http://scielo.iec.pa.gov.br

[28] Monteiro F, Schmidt ST, Costa IB, Almeida CCB, Matuda NS. Bolsa Família: insegurança alimentar e nutricional de crianças menores de cinco anos. Ciênc. Saúde Coletiva [internet]. 2014 [acesso em 26 maio 2017];19(5):1347-1358. Disponível em: http://www.scielo.br

[29] Cabral MJ, Vieira KA, Sawaya AL, Toledo TMM. Perfil socioeconômico, nutricional e de ingestão alimentar de beneficiários do Programa Bolsa Família. Estud. Avanç. [internet]. 2013 [acesso em 26 maio 2017];27(71):87-97. Disponível em: http://www.scielo.br 